\title{
Fast Prediction Of Indoor Pollutant Dispersion Based On
}

\section{Reduced-Order Ventilation Models}

Keywords: Indoor Ventilation, Decay, Reduced-order Models, Eigenmodes

\begin{abstract}
In order to provide realistic ventilation flow rates, it is of great importance to know indoor pollutant concentration field quickly and efficiently resulting from any type of indoorpollutant-source distribution, further facilitating the design and control of indoor ventilation systems for practical application. This work introduces the development of reduced-order ventilation models for transient pollutant dispersion. In particular, we focuss on transients resulting from a step change in pollutant source distributions. We further focuss on the decay problem. A reduced-order ventilation model is the solution for this decay problem, which is derived from a large coupled system of Ordinary Differential Equations (ODEs) for concentration that can be cast in terms of a matrix exponential, that is accurately represented with only a few dominant eigenmodes. Using a 2D ventilation case, dominant eigenmodes with their physical relevance and pollutant concentration results are presented. We find that the first 4 eigenmodes are sufficient to predict the pollutant concentration decay for the current test case. We also find that the complex eigenmodes play an important role in the indoor recirculation processes.
\end{abstract}




\section{INTRODUCTION}

In real life, mechanical ventilation systems are recommended to provide an acceptable level of supplied ventilation rates based on the product of designed occupants levels and the ventilation requirement per person. However, during a given day, ventilation systems are still operated based on the designed occupants levels instead of actual levels based on monitoring, often resulting in a waste of energy (Emmerich and Persily, 2001). In order to provide realistic ventilation flow rates, it is of great importance to know indoor pollutant concentration field quickly and efficiently resulting from any type of indoor-pollutant-source distribution. The intention of using reduced-order ventilation models is to predict pollutant concentration based on several dominant eigenmodes, i.e, eigenvalue and eigenvector pairs, which saves lots of computation effort, further facilitating the design and control of indoor ventilation systems for practical application.

The "reduced-order" model is initiated for fast prediction of pollutant dispersion under steady state indoor airflow. Indoor airflow is turbulent at first when the fan is operated, and it will be in the trend of stationary state after certain period, considered as steady airflow in this study. For the prediction of the indoor pollutant concentration with stationary source conditions, the low-dimensional linear ventilation models (cf. Cao and Meyers, 2012, 2013) have been developed. In this study, we develop reduced-order models for transient pollutant dispersion, with a focuss on the decay problem. Then, we formulate the solution in terms of a matrix exponential based on finite volume methods, and show that its representation can be formulated to only a few dominant eigenmodes. In Sec. 2, a reduced-order ventilation model is derived. In Sec. 3, the methodology is demonstrated on a simple 2D ventilation test case. Conclusions are drawn in Sec. 4. 


\section{DERIVATION OF A REDUCED-ORDER POLLUTANT-DECAY MODEL}

In the present work, we focuss on the transient cases, particulally on the elaboration of a representation of the decay problem. Consider the following concentration $c$ tansport equation for the decay problem,

$\frac{\partial c}{\partial t}+\nabla \cdot(u c)-\nabla \cdot(D \nabla c)=0, \quad x \in \Omega ; t \geq 0$

$c(x, 0)=c_{0}(x)$,

where $c_{0}$ is the initial concentration. Without loss of generality, we presume in the current section that $c_{0}(x) \geq 0$, so that also $c(x, t) \geq 0$.

The spatial discretization of Eq. (2.1), e.g., using a finite-volume approach, leads to a large coupled system of Ordinary Differential Equations (ODEs) that can be cast in following form,

$$
\begin{gathered}
\frac{\partial c}{\partial t}+\boldsymbol{A} \cdot c=0, \\
c(0)=c_{0} .
\end{gathered}
$$

Here $c \in P^{n}$ is a long vector containing the discrete concentrations at every cell in the solution domain (with $n$ cells). Further, $A \in P^{n \times n}$ is a matrix that represents the discretization of the operator $-(\nabla \cdot u-\nabla \cdot D \nabla)$, including the spatial boundary conditions (Ferziger and Peric, 2002). If appropriate spatial boundary conditions are used in Eq. (2.2), the matrix $\boldsymbol{A}$ is nonsingular, i.e. a valid set of boundary conditions leads to a unique solution of the stationary system. 
The solution of a linear system of first-order ODEs such as Eq. (2.2) can now be expressed using the matrix exponential $\exp (A t)$. To further elaborate this, $\boldsymbol{A}$ is diagonalized. To that end, we presume the matrix $A$ has $n$ linearly independent eigenvectors (Boyce and DiPrima,1992) and (Ockendon et al. (1999)). Thus, $\boldsymbol{A}=\boldsymbol{V} \Lambda \boldsymbol{V}^{-1}$, with $\Lambda$ a diagonal matrix containing the eigenvalues $\lambda_{j}$ of $\boldsymbol{A}$, and $\boldsymbol{V}$ a matrix of which the columns are the eigenvectors $v_{j}(j=1 \cdots n)$ of $A$. Further, the rows of $V^{-1}$ are the eigenvectors $v_{j}^{*}$ of $A^{T}$, i.e. it is easy to verify that $\boldsymbol{A}^{T}\left(\boldsymbol{V}^{-1}\right)^{T}=\Lambda\left(\boldsymbol{V}^{-1}\right)^{T}$, and the vectors $v_{j}$, and $v_{j}^{*}$ form a biorthogonal basis, i.e. $v_{i}^{T} v_{j}^{*}=\delta_{i j}$ (Boyce and DiPrima,1992). (Note that for symmetric matrices, $v_{j}=v_{j}^{*}$, but since the convection operator is non-symmetric, $A$ will also be a non-symmetric matrix.) Using these definitions, the solution to Eq. (2.2) is expressed as (Boyce and DiPrima,1992) and (Gockenbach, 2010)

$$
c(t)=\exp (A t) c_{0}=V \exp (\Lambda t) V^{-1} c_{0}=\sum_{j=1}^{n}\left(v_{j}^{*}{ }^{T} c_{0}\right) \exp \left(\lambda_{j} t\right) v_{j}
$$

In the context of three-dimensional dispersion simulations, with domains that contain hundred thousands or millions of cells $\left(n=10^{5}-10^{6}\right)$, it is not feasible to use Eq. (2.3) directly to solve Eq. (2.2), since the eigenvalue decomposition of $\boldsymbol{A}$ and $\boldsymbol{A}^{T}$ would require excessive computational times, proportional to $n^{3}$. However, Eq. (2.3) serves as an interesting starting point to construct a low-dimensional model. To that end, it is important to realize that Eq. (2.3) is a decay problem, so that $c \rightarrow 0$ for $t \rightarrow \infty$. Thus, the real parts of all eigenvalues of $A$ are negative, and can be arranged in decreasing order, such that $0>\lambda_{r, 1} \geq \lambda_{r, 2} \geq \lambda_{r, 3} \geq \cdots$.

The construction of a reduced-order pollutant-decay model now proceeds by truncating Eq.(2.3) to the first $q$ terms, i.e.

$$
c(t) \approx \sum_{j=1}^{q} a_{j} \exp \left(\lambda_{j} t\right) v_{j},
$$


with $a_{j}=\left(v_{j}^{* T} c_{0}\right)$. This requires $t$ to be sufficiently large such that $a_{q+j} \exp \left(\lambda_{q+j} t\right)\left\|V_{q+j}\right\| \approx 0$ $(j \geq 1)$. Hereby, we need to point out that the coefficients $a_{j}$ contains both real and complex values, which always appear in conjugate pairs.

\section{RESULTS AND DISCUSSION}

To test the methodology proposed in Section 2, we perform a RANS simulation of a simple two-dimensional ventilation benchmark case, using the open-source CFD software OpenFOAM. A detail of the geometry, and the computational mesh used for the CFD is provided in Fig. 1.

The computational mesh is block hexahedral structured (cf. Fig. 1) containing a total of 34000 cells (i.e. $160 \times 190$ in the main block, and $60 \times 60$ cells in the outlet). The slot-Reynolds number $R e_{s}$ (defined using inlet velocity $U_{0}$, and the inlet-slot height $0.6 \mathrm{~m}$ ) corresponds to 4200. At the inlet, a uniform velocity profile is applied with $U_{0}=0.4 \mathrm{~m} / \mathrm{s}$. The turbulence intensity is set at $10 \%$, and a turbulent length scale of $0.02 \mathrm{~m}$ is chosen. The non-dimensional ventilation equations are solved in RANS formulation, and closed with a low-Reynolds number $k--\varepsilon$ model (Chang et al. 1995), which shows the best aggreement with the experiments for the transional Reynolds number cases (Cao and Meyers, 2013) and (van Hooff et al.).

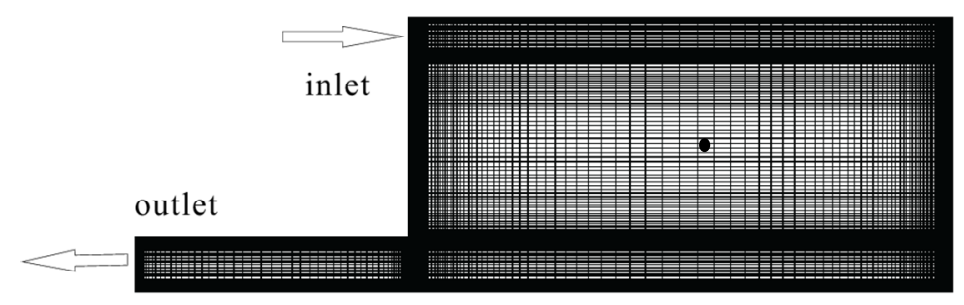

Figure 1. Computational setup of the indoor-ventilation test case 
Two RANS simulations are performed. First of all, a stationary simulation is executed that determines the flow solution and the initial concentration profile. To this end, a point source is used located at $(x, y)=(4.5,2.0)$ (cf. black dot in Fig.1). Secondly, a pollutant-decay simulation is executed starting from the initial concentration profile with the stationary background flow. The second simulation is used in Section 3.2 as a reference for concentration with the low-dimensional model. We will discuss eigenvalues and eigenmodes of the low-dimensional model in the next section.

\subsection{Discussion of largest eigenvalues and eigenvectors}

In the current section, we present the dominant eigenmodes associated with the selected test case, and discuss their physical relevance. Based on the velocity and the turbulent diffusion field, obtained from the stationary flow solution, the matrix $\boldsymbol{A}$ is now generated (cf. Eq. 2.2). Next, to obtain the eigenvalues and eigenmodes of $\boldsymbol{A}$, a parallel C code is written, that reads $A$ from disk, and employs the SLEPc library (Hernandez et al. 2005) for the eigenvalue analysis. In particular, the 'shift and invert' algorithm is used (Campos et al. 2012), that is specifically designed for the determination of the dominant eigenvalues and eigenvectors of large sparse matrices. For the current 2D case (with $\boldsymbol{A}$ a 30400 by 30400 matrix) we determine the first 14 dominant eigenmodes. This takes 2 min on 1 Xeon 5650 node with 8 cores, which is much less expensive compared to 2 hours with RANS simulation.

\subsection{Discussion on the largest eigenvalues}

Fig. 2 shows the 14 largest eigenvalues of the matrix $A$ in the complex plane. Apart from eigenvalues that are real, we also find complex eigenvalues. These come in complex conjugate pairs, and in Fig. 2, we explicitly accounted for that by giving them numbers $j(a)$, $j(b)$ respectively (e.g., $j=3$ ). As discussed earlier, only the real part of eigenvalues 
corresponds to the concentration decay. The larger the real part (closer to zero), the slower the corresponding eigenvector decays. From Fig. 2 it is obvious that the magnitude of eigenvalues' real part is negative. The corresponding eigenvectors are now further discussed.

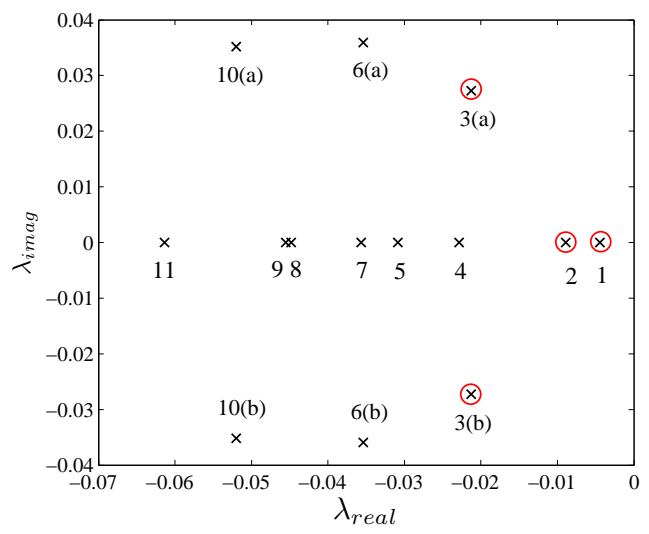

Figure 2. The 14 largest eigenvalues of matrix $A$ in the complex plane

\subsection{The first eigenmode}

In Fig. 3, we plotted the velocity magnitude, and the total diffusivity. In particular in regions where both velocity and diffusion are small, we will find high values for the first eigenmode (cf. Fig. 4). In these regions, pollution decays the slowest.

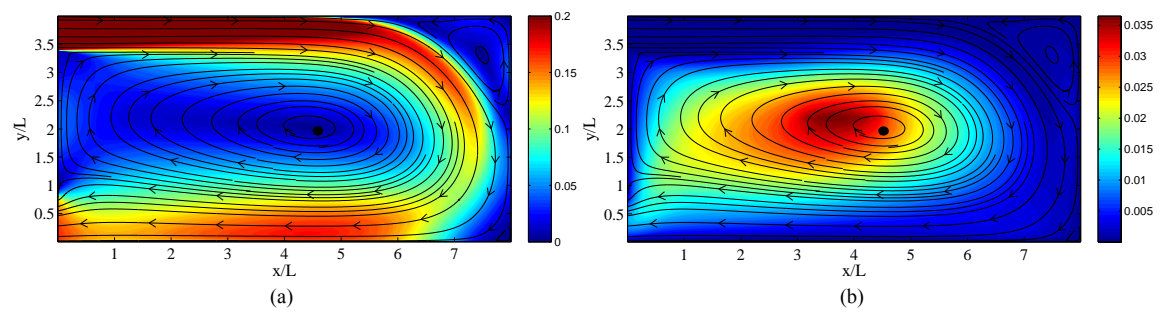

Figure 3. Illustration of streamlines, and distribution of velocity (a) and turbulent viscosity

(b) pollutant point source ()

We then focuss on the first eigenmode that represents the concentration distribution at a infinite time. In Fig. 4 (a), this eigenmode is displayed in color, and normalized by its largest 
value. In Figure 4, we also plotted the streamlines of the flow field. It is very clear that this flow field impacts on the shape of the eigenvector. Finally, we emphasize that the first eigenmode as shown in Fig. 4 (a) is positive. This is a requirement since for $c_{0}>0$ and $t \rightarrow \infty$, $c_{0}>0$ should hold.

\subsection{Real eigenmodes}

We further focuss on eigenmodes containing a real part only, as seen in Fig. 4 (a), (b), (e) and (f), representing the $2^{\text {nd }}, 4^{\text {th }}$ and $5^{\text {th }}$ dominant eigenmodes (corresponding to the eigenvalues shown in Fig. 4). Each of them are normalized by the largest absolute value of its corresponding eigenvector. Unlike the first eigenmode, higher modes can have both positive and negative values. However, given $c_{0}>0$, the sum of all eigenmodes need to be higher than 0 at any time. Thus the higher eigenmodes can be interpreted as corrections to the first eigenmode for $t<\infty$.

Fig. 4 (b) shows noticeable effects only in the upper corner of recirculation region. This is due to the fact that the turbulent mixing also happens along the flow trajectory between the primary recirculation and secondary recirculation region, thus potentially allowing for part of the pollutant to enter the secondary recirculation. This eigenmode further serves as a correction to the first eigenmode. 


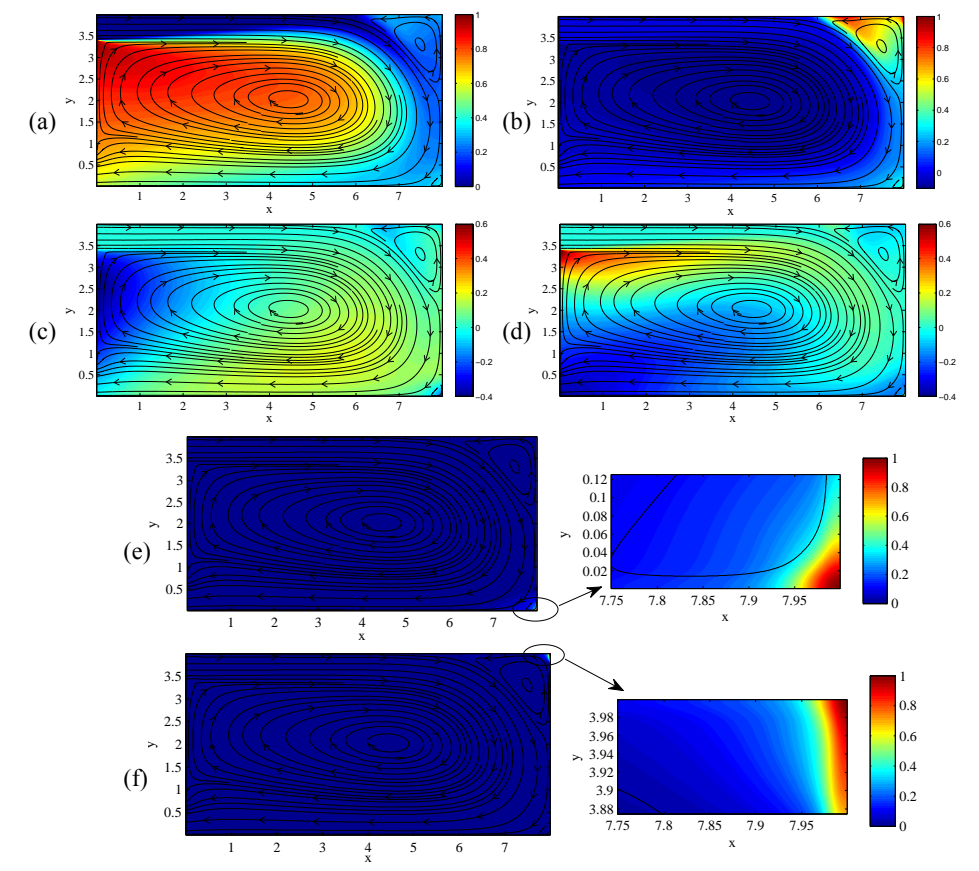

Figure 4. Streamlines and colour plot of 5 dominant eigenmodes, described by a set of normalized eigenvectors: [(a), (b), (e), (f): the $1^{s t}, 2^{\text {nd }}, 4^{\text {th }}, 5^{\text {th }}$ dominant eigenvectors, each normalized by the largest absolute value of its corresponding eigenvector; (c),(d): respectively corresponding to the real part and imaginary part of the 3(a) dominant eigenvector (a complex eigenmode), normalized by the the largest value of its eigenvector; the 3(b) eigenmode and the 3(a) eigenmode are a complex conjugate pair, so not shown here]

In Fig. 4 (e) and (f) (corresponding to the $4^{\text {th }}$ and $5^{\text {th }}$ eigenmodes), we observe that the eigenmode is prominent only in a very small corner region as shown by the zooms of Fig. 4 (e) and (f). We conclude that these two eigenmodes play a negligible role in the dispersion problem. We also investigate several higher eigenmodes, and similar behavior occurs.

Thus the overall conclusion is that the first 4 dominant eigenmodes $(q=4$, cf. Sec. 2$)$ are sufficient to estimate the pollutant concentration decay process for this $2 \mathrm{D}$ test case. The influence of the complex eigenmode is further discussed below. 


\subsection{Complex eigenmodes}

After considering the eigenmodes having only a real part, we now look at the eigenmodes having both a real part and imaginary part. The complex eigenmodes 3(a) and 3(b) (in Fig. 5) represent a complex conjugate pair. They share the same magnitude of the real part and imaginary part but having opposite signs for the imaginary part. Only one of the eigenmodes, i.e., 3(a) is considered. The real part and imaginary part of this eigenmode are shown in Fig. 5(c) and (d), normalized by the largest value of its eigenvector.

(a)

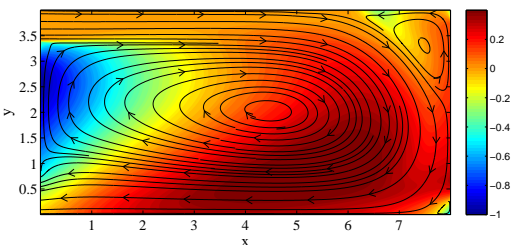

(c)

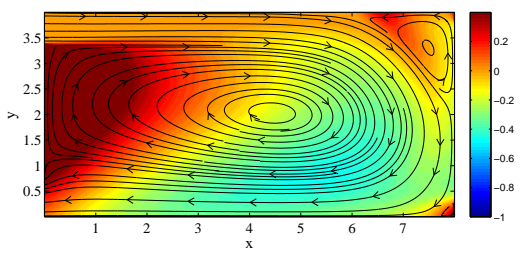

(b)

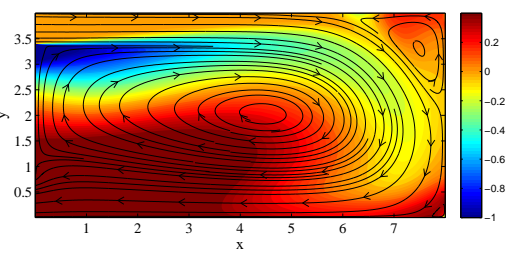

(d)

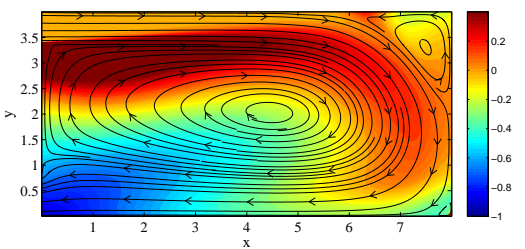

Figure 5. Streamlines and the periodic cycle of the complex eigenmodes (corresponding eigenvalues 3(a) and 3(b) shown in Fig. 2) shown for one period and with phase increments of $\pi / 2$

To understand Fig. 5(c) and (d) in detail, we revisit the knowledge of complex eigenvalues. Since $A$ is a real matrix, we recall that the complex eigen modes (eigen-solutions) always appear as complex conjugate pairs, defined as, $v_{j(a)}=\alpha+i \beta, v_{j(b)}=\alpha-i \beta$ the corresponding eigen values are represented as $\lambda_{j(a)}=\lambda_{r}+i \lambda_{i} \lambda_{j(b)}=\lambda_{r}-i \lambda_{i}$; the corresponding coefficients are denoted as $a_{j(a)}=a+i b, a_{j(b)}=a-i b$; where $\alpha, \lambda_{r}, a$ are the real parts of the complex numbers, and $\beta, \lambda_{r}, b$ are the imaginary parts. Thus the sum of a complex conjugate pair in Eq.(6.7) corresponds to $\left[a_{j(a)} \exp \left(\lambda_{j(a)} t\right) v_{j(a)}+a_{j(b)} \exp \left(\lambda_{j(b)} t\right) v_{j(b)}\right]$ 
$a_{j(a)} \exp \left(\lambda_{j(a)} t\right) v_{j(a)}+a_{j(b)} \exp \left(\lambda_{j(b)} t\right) v_{j(b)}$

$=2 \exp \left(\lambda_{r} t\right)\left[(a \alpha-b \beta) \cos \lambda_{i} t-(a \beta+b \alpha) \sin \lambda_{i} t\right]$

$=2 \exp \left(\lambda_{r} t\right)\left|a_{j}\right|\left|v_{j}\right| \mid \sin \left(\lambda_{i} t-\Phi\right)$,

where angular part $\Phi$ given by $\Phi=\arctan t((a \beta+b \alpha) /(a \alpha-b \beta))$. The above equation is the effect of exponential decay and sinusoidal phase function. Within this the larger the angle $\Phi$, the higher the frequency of rotation. We then expect to see periodic behaviour with a characteristic period or frequency in systems. Fig. 5 represents the periodic cycle corresponded with the complex eigen modes for 3(a) and 3(b) within a period of $\pi / 2$. We can clearly see that sinusoidal phase function participate only in the recirculation process and does not take part in overall concentration decay.

\section{CONCLUSIONS AND FUTURE WORK}

To test the current approach, a simple 2D ventilation case was considered. Using this case, two RANS simulations were performed. At first we conducted a stationary source simulation that determined the flow solution and the initial concentration profile. Based on the stationary background flow, we performed a pollutant-decay simulation starting from the initial concentration field. The second simulation was used as a reference for concentration with our reduced-order models. Later we discussed the impacts of the first few dominant eigenmodes (including both real and complex eigenmodes). We found that the first 4 eigenmodes are sufficient to predict the pollutant concentration decay particularly for this $2 \mathrm{D}$ case. We also found that the complex eigenmodes play a very important role in primary recirculation processes. To use reduced-order ventilation models, pollutant transport equation should be solved independently from Navier-stokes equation. This can be trivil sometimes due to the effects of density differences between air and pollutant, temperature influence. This will be future work of study. Further the low-dimensional reduced-order dispersion models will be studied based on the previous work (cf. Cao and Meyers, 2012), and we believe that 
reduced-order models may play an important role in on-line monitoring and control for ventilation systems. Moreover, for the study of reduced-order models for transient systems (specially as a decay problem), the CFD simulations was based on 2D cases. Therefore, cases in three dimensions and with more complex geometries for real life ventilation scenarios will be studied.

\section{ACKNOWLEDGEMENT}

The authors acknowledge financial support by the Flemish Science Foundation (FWO) with the grant number G.0435.08.

\section{REFERENCES}

Boyce W.E. and DiPrima R.C., (1992). Elementary Differential Equations. $5^{\text {th }}$ edn, New York: Wiley.

Cao, S.-J. and Meyers, J., (2012). On the construction and use of linear low-dimensional ventilation models. Indoor Air, 220 (5), 053 -- 64.

Cao, S.-J. and Meyers, J., (2013). Influence of turbulent boundary conditions on RANS simulations of pollutant dispersion in mechanically ventilated enclosures with transitional slot Reynolds number. Building and Environment, 590, 0397 -- 407.

Cao, S.-J. and Meyers, J., (2013). Asymptotic conditions for the use of linear ventilation models in the presence of buoyancy forces. Building Simulation.

Campos, C., Roman, J.E., Romero E. and Tomas. A., (2012). SLEPc Users Manual. Tech. Rep., DSIC-II/24/02 - Revision 3.3, Universitat Politècnica de València, 2012.

Chang, k.c., Hsieh, w.d., and Chen c.s., (1995). a modified low-reynolds-number turbulence model applicable to recirculating flow in pipe expansion. journal of fluids engineering, 1170 (3), 0 417---423. 
Emmerich S.J., Persily A.K., (2001). State-of-the-art review of $\mathrm{CO}_{2}$-based demand controlled ventilation technology and application. NISTIR 6729.

Ferziger, J.H., Peric,M., 2002. Computational Methods for Fluid Dynamics. Springer, London.

Gockenbach, M.S.\&Philadelphia, (2010). Partial Differential Equations. Society for Industrial and Applied Mathematics, 2 edition.

Hernandez V., Roman J. E. and Vidal V., (2005). SLEPc: A scalable and flexible toolkit for the solution of eigenvalue problems. ACM Trans. Math. Software, 310 (3), 0 351--362.

van Hooff T, Blocken B, van Heijst GJF, 2013. On the suitability of steady RANS CFD for forced mixing ventilation at transitional slot Reynolds numbers. Indoor Air 23, 236-249.

Ockendon, J., Howison, S., Lacey, A., and Movchan, A., 1999. Applied Partial Differential Equations. Oxford University Press. 